\title{
Necessity and Ethics of History Taking
}

\author{
Xuehong Wan and Rui Zeng
}

History taking is the first step of seeing a patient and serves as an important way to build up rapport between patients and doctors, through which doctors can help relieve patients from fear or misunderstanding of the disease, build up patients' trust in doctors to cure the disease, and enhance the patients' medical compliance.

Medical ethic belongs to professional ethics and involves many facets of clinical medicine. Regarding clinical diagnosis and treatment, it requires doctors to give the safest, least painful and most economical treatment for patients, no matter they are undergoing medical treatment, surgery or clinical trials.

1. Being serious and meticulous is a basic and key requirement, since only such attitude can build up confidence in patients to ensure good cooperation for acquiring a complete and accurate medical history. Doctors should be patient and attentive when listening to the patients.

2. Respect for the patient's privacy and ensuring confidentiality of all inquiry is an act of professionalism. Any information provided by the patient can only be used as scientific evidence for medical purposes. Doctors cannot disclose such information to others nor use it to embarrass or mock the patient.

3. Special attention should be given to the children and the elderly, considering they may not be able to report history fluently or respond properly to physicians as adults.

4. All patients should be treated equally, regardless of their economic, social or political status, education background, sex, age and race. Moreover, doctors should show more sympathy and consideration for the patients in difficult conditions. Discriminating against patients with disability is strictly prohibited.

5. Doctors should not criticize their colleagues at free will in front of patients, or show doubt and dissatisfaction toward past medical care the patient received. When other doctors are taking medical history, we should not make careless comments or evaluation, especially without knowledge of the full scope.

6. Doctors should also give the patients and their families as much health guidance and education as possible, which is part of the doctors' responsibility to the society.

X. Wan

Department of Internal medicine, Sichuan University,

Chengdu, China

R. Zeng $(\square)$

Department of Cardiology, Sichuan University, Chengdu, China 